\title{
Peri-Implantitis: Management of Ailing, Failing \& Failed Dental Implants
}

\author{
Dr. Ravi P. Popat ${ }^{1}$, Dr. Neeta V. Bhavsar ${ }^{1}$, Dr. Parita R. Popat ${ }^{2}$ \\ ${ }^{I}$ (Dept. of Peridontology, Govt. Dental College, Ahmedabad, India) \\ ${ }_{2}^{2}$ (Dept. of Conservative Dentistry \& Endodontics, Vaidik Dental College, Daman, India)
}

\begin{abstract}
Dental implants are widely used now-a-days for rehabilitation of partial \& complete edentulous. Although many literatures regarding dental implant reported to have long term success rate, failure of dental implant is not uncommon.Peri-implant disease is characterized by an inflammatory reaction in the tissues surrounding an implant. Peri-implant disease should be diagnosed \& treated as soon as possible to prevent implant failure. This literature review deals with various aspects ofperi-implant disease \& its management.
\end{abstract}

Keywords: Dental Implants, Peri-implant Diseases, Peri-implantitis, Peri-implant Pathology.

\section{Introduction}

Now-a-days use of dental implants play vital role in rehabilitation of partial \& complete edentulous subjects due to availability of wide range of treatment modalities. Although many literatures regarding dental implant reported to have long term success rate, they are not spared from complication \& failure. Implant failure occurs due to wide variety of reasons including improper treatment planning, surgical and prosthetic reason, material failure, and improper maintenance. Among them disease of soft \& hard tissue surrounding implant is presently major concern in implantology. This literature review deals with various aspects of peri-implant disease\& its management.

\section{Peri-implant disease}

Peri-implant diseases present in two forms;peri-implantmucositis and peri-implantitis. Both of theseare characterized by an inflammatory reaction inthe tissues surrounding an implant. Peri-implantmucositis has been described as a disease in whichthe presence of inflammation is confined to the soft tissues surrounding a dental implant with no signs of loss of supporting bone following initial bone remodelling during healing. Periimplantitis has been characterized by an inflammatory process around an implant, which includes both soft tissue inflammation and progressive loss of supporting bone beyond biological bone remodeling. ${ }^{1,2]}$

Distinct differences in the incidence and prevalenceof peri-implantitis have been reported by a numberof previous studies. In longitudinal study of 10 years including 101 subjects with 246 implants showed prevalence rate of periimplintitis $4.7 \%, 11.2 \%$ \& $15.1 \%$ respectively, among periodontally healthy, moderate \& severe periodontal compromised individuals. ${ }^{[3]} \mathrm{A}$ recent meta-analysis of nine studies with 1,497 participants and 6,283 implants estimated for the frequency of peri-implant mucositis were $63.4 \%$ of participants and $30.7 \%$ of implants, and those of peri-implantitis were $18.8 \%$ of participants and $9.6 \%$ of implants. A higher frequency of occurrence of peri-implant diseases was recorded for smokers. ${ }^{[4]}$ Findings show that peri-implant diseases are not uncommon following implant therapy.

\section{Ailing, failing \& failed implants}

Terminology of dental implant as ailing, failing, or failed is referring to the status of the peri-implant supporting tissues. A failed implant is one that is fractured, has been totally refractory to all methods of treatment, or demonstrates clinical mobility or circumferential periimplant radiolucency. These implants must be removed immediately, because progressive destruction of surrounding osseous tissues may occur. ${ }^{[5]}$

Ailing implant term was put forth during early 1990s in North American literature refers to clinically stable implant affected by bone loss withpocketing.A failing implant displays features similar to the ailing implant, but is refractory to therapy and continues to become worse. This implant also is immobile. ${ }^{[6,7]}$ According to other opinion implant referred to be ailing when affected by soft tissue aberrations without loss of supporting bone ${ }^{[8]}$ This definition seems synonyms with biologic complication can be defined as a soft tissue aberration without loss of the supporting bone. In general, problems limited to the soft tissue compartment and not involving the supportingbone defined as biologic complications or ailing implants. If the supporting bone is involved and the implant is still stable, the implant is failing. The implant is failed if it is mobile. ${ }^{[9]}$ 
However, there was difference in opinion regarding definition regarding ailing implant, the difference between ailing implant \& failing implant is distinct. The major difference between an ailing and a failing implant is the outcome of the therapy. In fact, if an ailing implant is resistant to therapy it becomes failing. In other words, the term ailing implies a somewhat more favourable prognosis than failing. ${ }^{[5,9]}$

\section{Management}

Before treatment of peri-implant pathology proper diagnosis of disease is required. Various diagnostic tools are as follows;

\subsection{Diagnosis of peri-implant pathology \\ 1.1.1. Peri-implant probing:}

Periodontal probing using a light probing force $(0.2-0.3 \mathrm{~N})$ is a reliable tool for diagnosing periimplant health and disease. In health the probe tip identified the apical extent of the barrier epithelium. Even mild inflammation around implants was associated with an increased probe penetration. Penetration of the probe up to $1.6 \mathrm{~mm}$ into the connective tissue occurred in the peri-implantitis lesion. Presence of bleeding on gentle probing $(0.25 \mathrm{~N})$ is a useful parameter for diagnosis of mucosal inflammation. Absence of bleeding on probing was an indicator for stable periimplant conditions. ${ }^{[10]}$

\subsubsection{Mobility:}

Mobility is not a good diagnostic as mobile implant is hopeless and should be removed. However,perceived implant mobility may be related to the loosened restorationand/or abutment, which may or may not lead to crestal bone loss withoutloss of integration. A loose implant-supported prosthesismay contribute to the accumulation of plaque, whichmay lead to the development of peri-implant diseases. ${ }^{[1]}$

\subsubsection{Radiographs:}

Periapical radiographs perpendicular to the implant body toshow a clear demarcation between the threads of theimplant. Other radiographs such as CBCT may beconsidered depending on the location of progressiveattachment loss. ${ }^{[1,10]}$

\subsubsection{Secondary Diagnostics:}

Bacterial culturing, inflammatory markers in peri-implant crevicular fluid\& saliva, and genetic diagnostics may beuseful in the diagnosis of peri-implant diseases. ${ }^{[1,10]}$

\subsection{Treatment strategies}

Treatment options for ailing and failing implants are varied from conservative to more aggressive therapy depending upon the situation. The overall goal of therapy is to arrest further loss of bone support, reestablish a healthy peri-implant mucosal seal\& to regenerate hard \& soft tissue to implant \& abutment. Treatment option varies according to aetiology. Treatment strategies are broadly divided in to nonsurgical \& surgical treatment strategies.

\subsubsection{Nonsurgical treatment:}

When biochemical forces of occlusion are considered as aetiological factor then,occlusal is first evaluated any occlusal interference if present, should be corrected.Fit of prosthesis \& abutment should also evaluated\& corrected if required.

When plaque \& microorganisms are suspected as aetiological factors, thorough debridement is treatment of choice.It was based on hypothesis that bacterial biofilms on implant surfacescause peri-implantitis, and that the removal of thesebacteria is the cure described by using the experimental gingivitis model originallydescribed by Loe et al. ${ }^{[11-13]}$ It was observed that mechanical non-surgical therapy could be effective in the treatment of peri-implant mucositislesions.mechanicalthe local debridement of hyperplastic peri-implant tissues using hand or ultrasonic plastic instruments has been suggested. Metallic instruments are avoided to minimize surface damages and roughening, which can favour plaque adhesion.Furthermore, the adjunctive use of antimicrobial mouth rinses enhanced the outcome of mechanical therapy of such mucositis lesions. Chemical disinfectants such as, hydrogen peroxide \&chlorhexidinedigluconate were used adjunct to mechanical debridement to improve clinical outcome. However, their results show variable findings. ${ }^{[14]}$

Local \& systemically administered antibiotics were used along with local debridement found to be effective in several cases. Local application of minocycline or doxycycline as an adjunct to mechanical debridement and irrigation with an antimicrobial agent may be effective in moderately deep lesions. The current available scientific information on the use of locally or systemically administered antibiotics is insufficient to allow any firm specific recommendations for the use of these drugs ${ }^{[15]}$ 


\subsubsection{Surgical treatment:}

In the instances, when condition is unstable after proper nonsurgical treatment, clinician should reevaluate the case \& may approach to surgical treatment if required. Surgical treatment includes the elevation of a mucoperiosteal flap and the removal of the peri-implant inflammatory granulation tissue.

Following surgical exposure of the contaminatedimplant surface mechanical,chemical, or photodynamic measuresand combinations of all three can beused to attempt to eliminate infection, resolve inflammation and render thesurface conducive to bone regenerationand re-osseointegration. Multiple procedures have been tried to decontaminate and condition the implant surface. Surfaces have been subjected to cleaning with carbon or plastic curettes, ultrasonic scalers, air-polishing devices using sodium bicarbonate or glycine powder, saline wash, peroxide, irradiation with hard or soft laser light, implantoplasty and/or the application of acids or various antimicrobial agents. However, there is no definitive gold standard method still proven. ${ }^{[16,17]}$

Various regenerative approaches including use of bone grafts \& use of barrier membrane had been documented. Goal of regenerative approach is to fill osseous defect around implant as well as to improve soft tissue condition. Autogenous bone, allogenic decalcified freeze-dried bone, xenogenic bone mineral, phytogenic calcium carbonate, hydroxyapatite or tri-calcium phosphate have been used in an attempt to fill peri-implant defects and regenerate bone. ePTFE, collagen, and resorbable synthetic membranes have been placed to cover the area. All of above documented method show various degree of success. Lack of properly conducted clinical trial does not allow for any firm specific recommendation of surgical therapy. ${ }^{[17]}$

\subsection{Peri-implant maintenance:}

According to the 2003 American Academy of Periodontology position paper, "patients should be evaluated at regular intervals to monitor their peri-implant status, the condition ofthe implant supported prostheses, and plaque control." Maintenance principles should include regular evaluation of implants and their surrounding tissues and prostheses; occlusal examination; review and reinforcement of oral hygiene; removal of plaque and calculus; treatment of disease or repair of prostheses, as required; and institution of customized preventive measures. ${ }^{[18]}$ Recent study showed the absence of preventive maintenance in individuals with preexisting peri-implant mucositis was associated with a high incidence of peri-implantitis. Clinical parameters, such as bleeding on peri-implant probing, periodontal probing depth and the presence of periodontitis were associated with a higher risk of developing peri-implantitis. ${ }^{[19]}$

\section{Conclusion}

Peri-implantitis is inflammatory disease of peri-implant tissue affecting osteo-integrated implant resulting eventually in implant failure. Treatment of peri-implant disease ranges from conventional nonsurgical therapy to aggressive surgical therapy. As treatment of peri-implantitis show variable rate of success, proper maintenance of peri-implant tissue is required for long term success of dental implants.

\section{References}

[1] Academy Report: Peri-Implant Mucositis and Peri-Implantitis: A Current Understanding of Their Diagnoses and Clinical Implications. Journal of Periodontology, 84(4), 2013, 436-443.

[2] M. Sanz, I.L. Chapple. Clinical research on peri-implant diseases: Consensus report of Working Group 4. Journal ofClinical Periodontology, 39(12s), 2012, 202-206.

[3] M. Roccuzzo, N. De Angelis, L. Bonino, M. Aglietta. Ten-year results of a three-arm prospective cohort study on implants in periodontally compromised patients. Part I: Implant loss and radiographic bone loss. ClinicalOral Implants Research, 21(5), 2010, 490-496.

[4] M.A. Atieh, N.H. Alsabeeha, C.M. Faggion Jr., W.J. Duncan. The frequency of peri-implant diseases: a systematic review and metaanalysis.Journal of Periodontology, 84(11), 2013, 1586-1598.

[5] M. Zablotsky, J. kwan. Periimplantitis: Etiology of the Ailing, Failing, or Failed Dental Implant. In W.B. Hall (Ed.), Critical Decision in Periodontology, (London: BC Decker, 2003) 256-257.

[6] J.Y. Kwan. The ailing implant.Journal ofthe California Dental Association, 19(12), 1991, 51-56.

[7] R.M. Meffert. How to treat ailing and failing implants.Implant Dentistry, 1(1), 1992, 25-33.

[8] J.T. Krauser. Hydroxylapatite-coated dental implants.Biologic rationale and surgical technique.Dental Clinics of North America, 33(4), 1989, 879-903.

[9] M. Esposito, J. Hirsch, U. Lekholm, P. Thomsen. Differential diagnosis and treatment strategies for biologic complications and failing oral implants: a review of the literature. The International Journal of Oral \&Maxillofacial Implants, 14(4), 1999, 473-490.

[10] L.J.A. Heitz-Mayfield. Peri-implant diseases: diagnosis and risk indicators. Journal of Clinical Periodontology, 35(8s), 2008, 292304.

[11] R. Pontoriero, M.P. Tonelli, G. Carnevale, A. Mombelli, S.R. Nyman, N.P. Lang. Experimentally induced peri-implant mucositis. A clinical study in humans.Clinical Oral Implants Research, 5(4), 1994, 254-259.

[12] N.U. Zitzmann, T. Berglundh, C.P. Marinello, J. Lindhe. Experimental peri-implant mucositis in man.Journal of Clinical Periodontology, 28(6), 2001, 517-523

[13] H. Loe, E. Theilade, S.B. Jensen. Experimental gingivitis in man.Journal of Periodontology, 36, 1965, $177-187$.

[14] S. Renvert, A.M. Roos-Jansaker, N. Claffey. Non-surgical treatment of peri-implant mucositis and peri-implantitis: a literature review. Journal of Clinical Periodontology, 35(8s), 2008, 305-315.

[15] A.J. Winkelhoff. Antibiotics in the treatment of peri-implantitis.European Journal of Oral Implantology, 5s, 2012, 43-50. 
[16] N. Claffey, E. Clarke, I. Polyzois, S. Renvert.Surgical treatment of peri-implantitis.Journal of Clinical Periodontology, 35s, 2008, :316-332.

[17] A.Mombelli, R.Moene, F.Decaillet. Surgical treatments of peri-implantitis.EuropeanJournal of Oral Implantology, 5s, 2012, 61-70.

[18] R.E.Cohen.Research, Science and Therapy Committee, American Academy of Periodontology. Position paper: Periodontal Maintenance. Journal of Periodontology, 74(9), 2003, 1395-1401.

[19] F.O. Costa, S. Takenaka-Martinez, L.O. Cota, S.D. Ferreira, G.L. Silva, J.E. Costa. Peri-implant disease in subjects with and without preventive maintenance: a 5-year follow-up. Journal of Clinical Periodontology, 39(2), 2012, 173-181. 